\title{
Periastron shift in Weyl class spacetimes
}

\author{
Donato Bini*§ $^{\llbracket}$, Francesco De Paolis ${ }^{\dagger}$, Andrea Geralico ${ }^{\dagger \S}$, \\ Gabriele Ingrosso ${ }^{\dagger}$ and Achille Nucita $^{\dagger}$ \\ * Istituto per le Applicazioni del Calcolo "M. Picone", CNR I-00161 Rome, Italy \\ $\S$ International Center for Relativistic Astrophysics - I.C.R.A., \\ University of Rome "La Sapienza", I-00185 Rome, Italy \\ ๆ INFN - Sezione di Firenze, Polo Scientifico, via Sansone, 1 \\ I-50019, Sesto Fiorentino (FI), Italy \\ $\dagger$ Dipartimento di Fisica, Università di Lecce, and INFN - Sezione di Lecce, \\ Via Arnesano, CP 193, I-73100 Lecce, Italy
}

July 13, 2018

\begin{abstract}
The periastron position advance for geodesic motion in axially symmetric solutions of the Einstein field equations belonging to the Weyl class of vacuum solutions is investigated. Explicit examples corresponding to either static solutions (single Chazy-Curzon, Schwarzschild and a pair of them), or stationary solution (single rotating Chazy-Curzon and Kerr black hole) are discussed. The results are then applied to the case of S2-SgrA* binary system of which the periastron position advance will be soon measured with a great accuracy.
\end{abstract}

Keywords: Periastron shift, Weyl class spacetimes.

PACS number: 04.20.Cv

\section{Introduction}

Stellar sources close enough to a massive central body will likely not be on simple Keplerian orbit due to general relativistic effects. In particular, a periastron position shift will result in (observable) rosetta shaped orbits. Since the amount of periastron advance strongly depends on the compactness of the central body, the detection of such an effect will give information about the nature of the central body itself. This could be exactly the case of stars orbiting close to the center of our Galaxy, where a "dark object" is presumably hosted. All the literature concerning this topic considers the central body as a static Schwarzschild black hole and a wide variety of related results as well as estimates for measurable shift effects can be found.

However, General Relativity contains other interesting exact solutions representing naked singularities or superposition of two or more axially symmetric bodies kept apart on stable configuration by gravitationally inert singular structures whose observational aspect have been poorly examined or often dismissed as representative of non-physical situations. On the other hand, singularities are somehow typical in general relativity and it is especially in order to better understand their role and their proper 
character in this quite simple class of solutions that the present paper has been conceived. In particular, together with the well known Schwarzschild black hole solution one may consider the Chazy-Curzon solution which represents the static exterior gravitational field of a deformed mass endowed with a naked singularity at the particle position 1, 2, Analogously, for the rotating case, together with the well known Kerr black hole solution one may consider the rotating Chazy-Curzon case.

Furthermore, due to a proper use of the Weyl formalism, it is easy to extend the discussion including the superposition of two or more axially symmetric bodies (in particular, two non-rotating ChazyCurzon particles or Schwarzschild black holes) aligned along the $z$-axis in a static configuration. These solutions are characterized by the occurrence of a conical singularity on the $z$-axis, which can be thought as a "strut", keeping the two bodies apart at a fixed distance (see also the pioneering works of Semerák, Zellerin and Žáček [3] 4] which considered accretion disks or rings of astrophysical interest).

The aim of the paper is to estimate the periastron shift of a star orbiting one of the above mentioned object and compare the results obtained. In doing so, we consider the S2-SgrA* binary system, hosted in the center of our Galaxy, which is of special interest in view of future observations possibly revealing a periastron shift effect.

The paper is structured as follows: in Section 2 we introduce some static metric in Weyl cylindrical coordinates: the single Chazy-Curzon particle, the single Schwarzschild black hole, two Chazy-Curzon particles and two Schwarzschild black holes, representing the gravitational field of a central body (a single mass located at the origin of the coordinates, or two masses displaced along the $z$-axis). In Section 3 we study the geodesic equations in such spacetimes in order to obtain a (linearized) expression for the periastron advance, up to the second order in a mass parameter associated to the central object (which exactly coincides with the mass if the central object is a single body; it is, instead, related to both the masses when the central object consists in a superposition of two bodies). In Section 4, we extend our treatment to the stationary case of a Kerr black hole as well as a rotating Chazy-Curzon particle. We apply our results to the case of S2-SgrA* binary system in Section 5, and in Section 6 we address some conclusions.

\section{Axisymmetric, static, vacuum solutions in Weyl formalism}

Axisymmetric, static, vacuum solutions of the Einstein field equations can be described by the Weyl formalism [5. The line element in cylindrical coordinates $(t, \rho, z, \phi)$ writes as

$$
\mathrm{d} s^{2}=-e^{2 \psi} \mathrm{d} t^{2}+e^{2(\gamma-\psi)}\left[\mathrm{d} \rho^{2}+\mathrm{d} z^{2}\right]+\rho^{2} e^{-2 \psi} \mathrm{d} \phi^{2},
$$

where the functions $\psi$ and $\gamma$ depend on the coordinates $\rho$ and $z$ only. The vacuum Einstein field equations in Weyl coordinates reduce to

$$
\begin{aligned}
0 & =\psi_{, \rho \rho}+\frac{1}{\rho} \psi_{, \rho}+\psi_{, z z} \\
0 & =\gamma_{, \rho}-\rho\left[\psi_{, \rho}^{2}-\psi_{, z}^{2}\right] \\
0 & =\gamma_{, z}-2 \rho \psi_{, \rho} \psi_{, z}
\end{aligned}
$$

The first equation is the three-dimensional Laplace equation written in cylindrical coordinates; so the function $\psi$ plays the role of a newtonian potential. The linearity of that equation allows to find explicit solutions representing superpositions of two or more axially symmetric bodies. In general, these solutions correspond to configurations not gravitationally stable because of the occurrence of gravitationally inert singular structures ("struts" and "membranes") that keep the bodies apart (see, e.g. [6] and references therein). Properly speaking, this is the effect of the presence of a conical 
singularity on the $z$-axis, the occurrence of which is related to the non-vanishing of the function $\gamma(\rho, z)$ on the portion of the axis between the sources or outside them.

For the static axisymmetric vacuum solutions the regularity condition on the axis of symmetry ("elementary flatness") is given by

$$
\lim _{\rho \rightarrow 0} \gamma=0 .
$$

Let us briefly summarize the properties of the solutions belonging to the Weyl class representing the field of a Chazy-Curzon particle or a Schwarzschild black hole as well as superpositions of two of them.

\section{The single Chazy-Curzon particle}

A single Chazy-Curzon particle is a static axisymmetric solution of Einstein's equations endowed with a naked singularity at the particle position 11, 2, 7. The Curzon metric is generated by the newtonian potential of a spherically symmetric point mass using the Weyl formalism:

$$
\psi_{\mathrm{C}}=-\frac{M_{\mathrm{C}}}{R_{\mathrm{C}}}, \quad \gamma_{\mathrm{C}}=-\frac{1}{2} \frac{M_{\mathrm{C}}^{2} \rho^{2}}{R_{\mathrm{C}}^{4}}, \quad R_{\mathrm{C}}=\sqrt{\rho^{2}+z^{2}} .
$$

\section{Superposition of two Chazy-Curzon particles}

The solution corresponding to the superposition of two Chazy-Curzon particles with masses $M_{\mathrm{C}}$ and $m_{\mathrm{C}_{\mathrm{b}}}$ and positions $z=0$ and $z=b$ on the $z$-axis respectively is given by metric (11) with functions

$$
\psi=\psi_{\mathrm{C}}+\psi_{\mathrm{C}_{\mathrm{b}}}, \quad \gamma=\gamma_{\mathrm{C}}+\gamma_{\mathrm{C}_{\mathrm{b}}}+\gamma_{\mathrm{CC}_{\mathrm{b}}},
$$

where $\psi_{\mathrm{C}}, \gamma_{\mathrm{C}}$ are defined by Eq. (4), while

$$
\psi_{\mathrm{C}_{\mathrm{b}}}=-\frac{m_{\mathrm{C}_{\mathrm{b}}}}{R_{\mathrm{C}_{\mathrm{b}}}}, \quad \gamma_{\mathrm{C}_{\mathrm{b}}}=-\frac{1}{2} \frac{m_{\mathrm{C}_{\mathrm{b}}}^{2} \rho^{2}}{R_{\mathrm{C}_{\mathrm{b}}}^{4}}, \quad R_{\mathrm{C}_{\mathrm{b}}}=\sqrt{\rho^{2}+(z-b)^{2}}
$$

and $\gamma_{\mathrm{CC}_{\mathrm{b}}}$ can be obtained by solving Einstein's equations (2):

$$
\gamma_{\mathrm{CC}_{\mathrm{b}}}=2 \frac{m_{\mathrm{C}_{\mathrm{b}}} M_{\mathrm{C}}}{b^{2}} \frac{\rho^{2}+z(z-b)}{R_{\mathrm{C}_{\mathrm{b}}} R_{\mathrm{C}}}+C .
$$

The value of the arbitrary constant $C$ can be determined by imposing the regularity condition (3). Note that in order to make the function $\gamma_{\mathrm{CC}_{\mathrm{b}}}$ vanishes on the whole $z$-axis, the constant value $C$ cannot be uniquely chosen. In fact, setting $\gamma_{\mathrm{CC}_{\mathrm{b}}} \neq 0$ gives rise to a conical singularity (see, e.g. 8, 9]), corresponding to a strut in compression, which holds the two particles apart. On the other hand, the choice $C=2 m_{\mathrm{C}_{\mathrm{b}}} M_{\mathrm{C}} / b^{2}$ makes $\gamma_{\mathrm{CC}_{\mathrm{b}}}=0$ only on the segment $0<z<b$ of the $z$-axis between the sources. In the following we use $C=-2 m_{\mathrm{C}_{\mathrm{b}}} M_{\mathrm{C}} / b^{2}$, that makes $\gamma_{\mathrm{CC}_{\mathrm{b}}}=0$ on the portion of the axis with $z<0$ and $z>b$.

\section{The single Schwarzschild black hole}

The Schwarzschild black hole solution in Weyl coordinates is generated by the newtonian potential of a line source (a homogeneous rod) of mass $M_{\mathrm{S}}$ and lenght $2 L$ (with the further position $L=M_{\mathrm{S}}$ ), lying on the axis and placed about the origin:

$$
\begin{aligned}
\psi_{\mathrm{S}} & =\frac{1}{2} \ln \left[\frac{R_{1}^{+}+R_{1}^{-}-2 M_{\mathrm{S}}}{R_{1}^{+}+R_{1}^{-}+2 M_{\mathrm{S}}}\right], \quad \gamma_{\mathrm{S}}=\frac{1}{2} \ln \left[\frac{\left(R_{1}^{+}+R_{1}^{-}\right)^{2}-4 M_{\mathrm{S}}^{2}}{4 R_{1}^{+} R_{1}^{-}}\right], \\
R_{1}^{ \pm} & =\sqrt{\rho^{2}+\left(z \pm M_{\mathrm{S}}\right)^{2}} .
\end{aligned}
$$


The usual line element in standard Boyer-Lindquist coordinates $(t, r, \theta, \phi)$ is recovered by making the following transformation:

$$
\rho=\sqrt{r^{2}-2 M_{\mathrm{S}} r} \sin \theta, \quad z=\left(r-M_{\mathrm{S}}\right) \cos \theta .
$$

\section{Superposition of two Schwarzschild black holes}

The solution corresponding to a linear superposition of two Schwarzschild black holes with masses $M_{\mathrm{S}}$ and $m_{\mathrm{S}_{\mathrm{b}}}$ and positions $z=0$ and $z=b$ on the $z$-axis respectively is given by metric (1) with functions

$$
\psi=\psi_{\mathrm{S}}+\psi_{\mathrm{S}_{\mathrm{b}}}, \quad \gamma=\gamma_{\mathrm{S}}+\gamma_{\mathrm{S}_{\mathrm{b}}}+\gamma_{\mathrm{SS}_{\mathrm{b}}},
$$

where $\psi_{\mathrm{S}}, \gamma_{\mathrm{S}}$ are defined by Eq. (8), while

$$
\begin{aligned}
\psi_{\mathrm{S}_{\mathrm{b}}} & =\frac{1}{2} \ln \left[\frac{R_{2}^{+}+R_{2}^{-}-2 m_{\mathrm{S}_{\mathrm{b}}}}{R_{2}^{+}+R_{2}^{-}+2 m_{\mathrm{S}_{\mathrm{b}}}}\right], \quad \gamma_{\mathrm{S}_{\mathrm{b}}}=\frac{1}{2} \ln \left[\frac{\left(R_{2}^{+}+R_{2}^{-}\right)^{2}-4 m_{\mathrm{S}_{\mathrm{b}}}^{2}}{4 R_{2}^{+} R_{2}^{-}}\right] \\
\gamma_{\mathrm{SS}_{\mathrm{b}}} & =\frac{1}{2} \ln \left[\frac{E_{\left(1^{+}, 2^{-}\right)} E_{\left(1^{-}, 2^{+}\right)}}{E_{\left(1^{+}, 2^{+}\right)} E_{\left(1^{-}, 2^{-}\right)}}\right]+C, \quad E_{\left(1^{ \pm}, 2^{ \pm}\right)}=\rho^{2}+R_{1}^{ \pm} R_{2}^{ \pm}+Z_{1}^{ \pm} Z_{2}^{ \pm} \\
R_{1}^{ \pm} & =\sqrt{\rho^{2}+\left(Z_{1}^{ \pm}\right)^{2}}, \quad R_{2}^{ \pm}=\sqrt{\rho^{2}+\left(Z_{2}^{ \pm}\right)^{2}} \\
Z_{1}^{ \pm} & =z \pm M_{\mathrm{S}}, \quad Z_{2}^{ \pm}=z-\left(b \mp m_{\mathrm{S}_{\mathrm{b}}}\right)
\end{aligned}
$$

the function $\gamma_{\mathrm{SS}_{\mathrm{b}}}$ being obtained by solving Einstein's equations (2). The value of arbitrary constant $C$ can be determined by imposing the regularity condition (3); we make the choice $C=0$, so that the function $\gamma_{\mathrm{SS}_{\mathrm{b}}}$ vanishes on the portions of the $z$-axis outside the sources (that is, for $z>b+m_{\mathrm{S}_{\mathrm{b}}}$ and $z<-M_{\mathrm{S}}$.

\section{Periastron shift of a distant orbiting star}

The geodesic motion of a test particle in a plane orthogonal to the $z$-axis (i.e. $z=$ const), from the metric (1) is described by the equations

$$
\begin{aligned}
0 & =\ddot{\phi}+\frac{2}{\rho}\left(1-\rho \psi_{, \rho}\right) \dot{\rho} \dot{\phi} \\
0 & =e^{-2(\gamma-2 \psi)}\left[\dot{t}^{2}+\rho^{2} e^{-4 \psi} \dot{\phi}^{2}\right] \psi_{, z}-\left[\gamma_{, z}-\psi, z\right] \dot{\rho}^{2} \\
0 & =e^{-2(\gamma-2 \psi)}\left[\psi_{, \rho} \dot{t}^{2}-\rho e^{-4 \psi}\left(1-\rho \psi_{, \rho}\right)\right] \dot{\phi}^{2}+\left[\gamma_{, \rho}-\psi_{, \rho}\right] \dot{\rho}^{2}+\ddot{\rho} \\
0 & =\ddot{t}+2 \psi_{, \rho} \dot{\rho} \dot{\rho},
\end{aligned}
$$

where the dot represents differentiation with respect to the orbit proper time parameter and the metric functions $\psi$ and $\gamma$ now depend only on $\rho$. When the $z=$ const plane is a symmetry plane (the $z=0$ plane for a single-body solution, or the middle plane in the case of two bodies of equal mass), this system is further simplified, since in this case $\psi_{, z}=0$, and so $\gamma_{, z}=0$ too.

Due to the Killing symmetries of the metric the conserved energy per unit mass $(E)$ and angular momentum per unit mass $(L)$ of the particle can be introduced:

$$
E=e^{2 \psi} \dot{t}, \quad L=\rho^{2} e^{-2 \psi} \dot{\phi} .
$$


Moreover, by using the timelike condition for the geodesic,

$$
-e^{-2 \psi} E^{2}+e^{2(\gamma-\psi)} \dot{\rho}^{2}+\frac{e^{2 \psi}}{\rho^{2}} L^{2}=-1
$$

the shape of the orbit (i.e. $\rho$ as a function of $\phi$ ) is described by

$$
\frac{\mathrm{d} \rho}{\mathrm{d} \phi}=\rho^{2} e^{-(\gamma+2 \psi)}\left[\frac{1}{B^{2}}-\frac{e^{4 \psi}}{\rho^{2}}-\frac{e^{2 \psi}}{L^{2}}\right]^{1 / 2},
$$

being $B=L / E$.

Let us assume the test particle bound in an elliptic orbit around the compact object (which may be a single Chazy-Curzon particle, a single Schwarzschild black hole or a pair of them) on a plane orthogonal to the $z$-axis. The orbit followed by the test particle can be parametrized as

$$
\rho=\frac{d\left(1-e^{2}\right)}{1+e \cos \chi}
$$

where $d$ and $e$ can be thought as the ellipse semi-major axis and eccentricity, and where $\chi$ is a new variable called relativistic anomaly. At periastron and aphastron, $\rho$ reaches its minimum $\left(\rho_{-}=d(1-e)\right)$ and maximum $\left(\rho_{+}=d(1+e)\right)$ values obtained from eq. (16) for $\chi=0$ and $\chi=\pi$ respectively. At these points $\mathrm{d} \rho / \mathrm{d} \phi$ vanishes so that eq. (15) gives

$$
\left[\frac{e^{4 \psi_{ \pm}}}{\rho_{ \pm}^{2}}-\frac{e^{2 \psi_{ \pm}}}{L^{2}}\right]=\frac{1}{B^{2}}
$$

with $\psi_{ \pm}=\psi\left(\rho_{ \pm}\right)$. From the two previous equations one can derive the two constant of motion to be

$$
B^{2}=\frac{e^{-2 \psi_{-}}-e^{-2 \psi_{+}}}{\left[\frac{e^{2 \psi_{-}}}{\rho_{-}^{2}}-\frac{e^{2 \psi_{+}}}{\rho_{+}^{2}}\right]}, \quad L^{2}=\left[\frac{e^{-2 \psi_{-}}}{B^{2}}-\frac{e^{2 \psi_{-}}}{\rho_{-}^{2}}\right]^{-1} .
$$

It is useful to express $\phi$ as a function of the relativistic anomaly $\chi$, so that

$$
\frac{\mathrm{d} \phi}{\mathrm{d} \chi}=\left.\frac{d\left(1-e^{2}\right) e \sin \chi}{(1+e \cos \chi)^{2}} \frac{\mathrm{d} \phi}{\mathrm{d} \rho}\right|_{\rho=\rho(\chi)}
$$

where $\mathrm{d} \phi / \mathrm{d} \rho$ can be derived from eq. (15) by using the relation $\rho=\rho(\chi)$ of eq. (16). The total change in $\phi$ as $\chi$ decreases from $\pi$ to 0 is the same as the change in $\phi$ as $\chi$ increases from 0 to $\pi$, so that the total change in $\phi$ per revolution is $2|\phi(\pi)-\phi(0)|$, where

$$
\phi(\pi)-\phi(0)=\left.\int_{0}^{\pi} \frac{d\left(1-e^{2}\right) e \sin \chi}{(1+e \cos \chi)^{2}} \frac{\mathrm{d} \phi}{\mathrm{d} \rho}\right|_{\rho=\rho(\chi)} \mathrm{d} \chi .
$$

This would equal $2 \pi$ if the orbit is a closed ellipse, so in general the orbit precesses by an angle

$$
\Delta \phi=2|\phi(\pi)-\phi(0)|-2 \pi
$$

per revolution. The integral appearing at the second hand in eq. (20) can be generally expressed in terms of elliptic integrals. However, by noting that the ratio between the total mass $M$ of the central 
object and the major axis $d$ of the considered orbit is a very small quantity, it is justifiable to expand the integral in powers of $M$, retaining only the first power.

We are interested here in the estimate of the differences in the periastron position advance due to the presence of a central massive object consisting in a single or two Chazy-Curzon particles or Schwarzschild black holes, in order to priviledge either configuration when a physical system (for instance the S2-SgrA* binary system) is investigated. As we shall see soon, all the considered solutions give rise to the same amount of periastron shift at first order in $M$, while differences occurr in the second order term. In the case of a single Chazy-Curzon particle and a Schwarzschild black hole, by using the metric functions (4) and (8) respectively evaluated at $z=0$, the integral (20) gives

$$
\begin{aligned}
\Delta \phi_{\mathrm{C}} & \simeq \frac{6 \pi M_{\mathrm{C}}}{d\left(1-e^{2}\right)}+\frac{\left(44-9 e^{2}\right) \pi M_{\mathrm{C}}^{2}}{2 d^{2}\left(1-e^{2}\right)^{2}} \\
\Delta \phi_{\mathrm{S}} & \simeq \frac{6 \pi M_{\mathrm{S}}}{d\left(1-e^{2}\right)}+\frac{3\left(14-3 e^{2}\right) \pi M_{\mathrm{S}}^{2}}{2 d^{2}\left(1-e^{2}\right)^{2}} .
\end{aligned}
$$

Therefore, the two cases differ for the second order term; if we take $M_{\mathrm{C}}=M=M_{\mathrm{S}}$, the difference reads out to be

$$
\Delta \phi_{\mathrm{C}}-\Delta \phi_{\mathrm{S}}=\frac{\pi M^{2}}{d^{2}\left(1-e^{2}\right)^{2}} \equiv \Delta .
$$

As far as a system of two axially symmetric bodies is concerned, we limit our analysis to the symmetric configuration consisting of two equal object endowed each with the same mass $M$ displaced along the $z$-axis and separated by a distance $b$. It has been demonstrated [10] that in this special case timelike circular geodesics exist on the middle plane $z=b / 2$. So we apply the above developed formalism to a test particle which orbits a system of two equal mass Chazy-Curzon singularities $\left(m_{\mathrm{C}_{\mathrm{b}}}=M_{\mathrm{C}}\right)$ or Schwarzschild black holes $\left(m_{\mathrm{S}_{\mathrm{b}}}=M_{\mathrm{S}}\right)$ on the middle plane. In both cases we solve the integral (20) by expanding the argument also in the distance parameter $b$ up to the second order. The expected periastron shift results to be

$$
\begin{aligned}
\Delta \phi_{\mathrm{CC}_{\mathrm{b}}} & \simeq \frac{12 \pi M_{\mathrm{C}}}{d\left(1-e^{2}\right)}+\frac{2\left(44-9 e^{2}\right) \pi M_{\mathrm{C}}^{2}}{d^{2}\left(1-e^{2}\right)^{2}}-\frac{3}{4} \frac{\pi b^{2}}{d^{2}\left(1-e^{2}\right)^{2}}, \\
\Delta \phi_{\mathrm{SS}_{\mathrm{b}}} & \simeq \frac{12 \pi M_{\mathrm{S}}}{d\left(1-e^{2}\right)}+\frac{3\left(29-6 e^{2}\right) \pi M_{\mathrm{S}}^{2}}{d^{2}\left(1-e^{2}\right)^{2}}-\frac{3}{4} \frac{\pi b^{2}}{d^{2}\left(1-e^{2}\right)^{2}} .
\end{aligned}
$$

Note that the solutions for a single Chazy-Curzon particle or Schwarzschild black hole in $z=0$ can be recovered from the previous relations by putting $b \rightarrow 0$ and $M \rightarrow M / 2$ in the first case and $b \rightarrow 2 M$ and then $M \rightarrow M / 2$ in the latter case, respectively. The difference in the periastron shift exactly coincides with that estimated for the corresponding single-body solutions:

$$
\Delta \phi_{\mathrm{CC}_{\mathrm{b}}}-\Delta \phi_{\mathrm{SS}_{\mathrm{b}}}=\Delta
$$

by taking $M_{\mathrm{C}}=M=M_{\mathrm{S}}$, with $\Delta$ defined by (23).

It is interesting to point out that the second order term appearing in both formulae (24) can be made vanishing by a suitable choice of the distance $b$ between the two bodies: by introducing the distance parameter $\beta=b / M$, we find

$$
\beta_{\mathrm{CC}_{\mathrm{b}}}=\frac{2}{3} \sqrt{6} \sqrt{44-9 e^{2}}, \quad \beta_{\mathrm{SS}_{\mathrm{b}}}=2 \sqrt{29-6 e^{2}} .
$$




\section{Axisymmetric, stationary, vacuum solutions in Weyl formal- ism}

The line element of a stationary axisymmetric spacetime is given by the Lewis-Papapetrou metric [1]

$$
\mathrm{d} s^{2}=-e^{2 \psi}[\mathrm{d} t-w \mathrm{~d} \phi]^{2}+e^{2(\gamma-\psi)}\left[\mathrm{d} \rho^{2}+\mathrm{d} z^{2}\right]+\rho^{2} e^{-2 \psi} \mathrm{d} \phi^{2}
$$

where the function $\psi, \gamma$ and $w$ depend on the coordinates $\rho$ and $z$ only. The vacuum Einstein field equations in Weyl coordinates reduce to

$$
\begin{aligned}
0 & =\psi_{, \rho \rho}+\frac{1}{\rho} \psi_{, \rho}+\psi_{, z z}+\frac{e^{4 \psi}}{2 \rho^{2}}\left[w_{, \rho}^{2}+w_{, z}^{2}\right], \\
0 & =w_{, \rho \rho}-\frac{1}{\rho} w_{, \rho}+w_{, z z}+4\left[w_{, \rho} \psi_{, \rho}+w_{, z} \psi_{, z}\right], \\
0 & =\gamma_{, \rho}-\rho\left[\psi_{, \rho}^{2}-\psi_{, z}^{2}\right]+\frac{e^{4 \psi}}{4 \rho}\left[w_{, \rho}^{2}-w_{, z}^{2}\right], \\
0 & =\gamma_{, z}-2 \rho \psi_{, \rho} \psi_{, z}+\frac{e^{4 \psi}}{2 \rho} w_{, \rho} w_{, z} .
\end{aligned}
$$

The metric function $\gamma$ is obtained by quadrature once the solutions $\psi$ and $w$ for the nonlinear coupled system of the first two equations above are known. The geodesic equations for the metric (27) (when the motion is confined on a $z=$ const plane) are given by

$$
\begin{aligned}
0= & \ddot{t}+\left[2 \psi_{, \rho}+\frac{e^{4 \psi}}{\rho^{2}} w w_{, \rho}\right] \dot{t} \dot{\rho}+\left[-4 \psi_{, \rho} w-\left(1+\frac{e^{4 \psi}}{\rho^{2}} w^{2}\right) w_{, \rho}+\frac{2}{\rho} w\right] \dot{\rho} \dot{\phi}, \\
0= & e^{-2(\gamma-2 \psi)}\left\{\psi_{, z} \dot{t}^{2}-\left(w_{, z}+2 w \psi, z\right) \dot{\phi} \dot{t}+\left[w\left(w_{, z}+w \psi, z\right)+e^{-4 \psi} \rho^{2} \psi, z\right] \dot{\phi}^{2}\right\} \\
& +\left[\psi_{, z}-\gamma_{, z}\right] \dot{\rho}^{2} \\
0= & e^{-2(\gamma-2 \psi)}\left\{\psi_{, \rho} \dot{t}^{2}-\left(w_{, \rho}+2 w \psi_{, \rho}\right) \dot{\phi} \dot{t}+\left[w\left(w_{, \rho}+w \psi_{, \rho}\right)-e^{-4 \psi} \rho\left(1-\rho \psi_{, \rho}\right)\right] \dot{\phi}^{2}\right\} \\
& +[\gamma, \rho-\psi, \rho] \dot{\rho}^{2}+\ddot{\rho}, \\
0 & \ddot{\phi}+\frac{e^{4 \psi}}{\rho^{2}} w_{, \rho} \dot{t} \dot{\rho}+\left[\frac{2}{\rho}-\frac{e^{4 \psi}}{\rho^{2}} w w_{, \rho}-2 \psi_{, \rho}\right] \dot{\rho} \dot{\phi} .
\end{aligned}
$$

Following the same procedure of the static case, from the previous set of equations we finally obtain

$$
\frac{\mathrm{d} \rho}{\mathrm{d} \phi}=\frac{e^{-(\gamma-2 \psi)}}{1-\frac{2 w}{B}}\left[e^{-4 \psi} \rho^{2}-w\left(e^{-2 \psi}-4 w\right)\right]\left[\frac{1}{B^{2}}-\frac{\left(1-\frac{2 w}{B}\right)^{2}}{e^{-4 \psi} \rho^{2}-w\left(e^{-2 \psi}-4 w\right)}-\frac{e^{2 \psi}}{L^{2}}\right]^{1 / 2}
$$

where the metric functions $\psi, \gamma$ and $w$ now depend only on $\rho$. The periastron shift is given again by eq. (21), by substituting the previous expression for $\mathrm{d} \rho / \mathrm{d} \phi$ into equation (20). As applications we shall consider next the solutions corresponding to the single Kerr black hole as well as the rotating Chazy-Curzon particle. Superposition of two rotating solutions can also be considered. However, the involved formulae are rather complicated, and we shall not pursue this extension here.

1. The Kerr black hole

The metric functions generating the Kerr black hole solution in Weyl coordinates are given by

$$
\psi_{\mathrm{K}}=\frac{1}{2} \ln \left[\frac{\left(R_{\mathrm{K}}^{+}+R_{\mathrm{K}}^{-}\right)^{2}-4 M_{\mathrm{K}}^{2}+\frac{a^{2}}{\sigma^{2}}\left(R_{\mathrm{K}}^{+}-R_{\mathrm{K}}^{-}\right)^{2}}{\left[R_{\mathrm{K}}^{+}+R_{\mathrm{K}}^{-}+2 M_{\mathrm{K}}\right]^{2}+\frac{a^{2}}{\sigma^{2}}\left(R_{\mathrm{K}}^{+}-R_{\mathrm{K}}^{-}\right)^{2}}\right],
$$




$$
\begin{aligned}
& \gamma_{\mathrm{K}}=\frac{1}{2} \ln \left[\frac{\left(R_{\mathrm{K}}^{+}+R_{\mathrm{K}}^{-}\right)^{2}-4 M_{\mathrm{K}}^{2}+\frac{a^{2}}{\sigma^{2}}\left(R_{\mathrm{K}}^{+}-R_{\mathrm{K}}^{-}\right)^{2}}{4 R_{\mathrm{K}}^{+} R_{\mathrm{K}}^{-}}\right], \\
& w_{\mathrm{K}}=-\frac{a M_{\mathrm{K}}}{\sigma^{2}} \frac{\left[R_{\mathrm{K}}^{+}+R_{\mathrm{K}}^{-}+2 M_{\mathrm{K}}\right]\left[\left(R_{\mathrm{K}}^{+}-R_{\mathrm{K}}^{-}\right)^{2}-4 \sigma^{2}\right]}{\left(R_{\mathrm{K}}^{+}+R_{\mathrm{K}}^{-}\right)^{2}-4 M_{\mathrm{K}}^{2}+\frac{a^{2}}{\sigma^{2}}\left(R_{\mathrm{K}}^{+}-R_{\mathrm{K}}^{-}\right)^{2}},
\end{aligned}
$$

where

$$
R_{\mathrm{K}}^{ \pm}=\sqrt{\rho^{2}+(z \pm \sigma)^{2}}, \quad \sigma=\sqrt{M_{\mathrm{K}}^{2}-a^{2}},
$$

$M_{\mathrm{K}}$ and $a$ being the mass and the specific angular momentum of the source, respectively. The usual line element in Boyer-Lindquist coordinates $(t, r, \theta, \phi)$ is recovered by making the transformation

$$
\rho=\sqrt{r^{2}-2 M_{\mathrm{K}} r+a^{2}} \sin \theta, \quad z=\left(r-M_{\mathrm{K}}\right) \cos \theta .
$$

Following the procedure described in the previous section, the periastron shift of a star orbiting a Kerr black hole is given by

$$
\Delta \phi_{\mathrm{K}} \simeq \frac{6 \pi M_{\mathrm{K}}}{d\left(1-e^{2}\right)}+\frac{16 a \pi M_{\mathrm{K}}^{1 / 2}}{d^{3 / 2}\left(1-e^{2}\right)^{3 / 2}}+\frac{3\left(14-3 e^{2}\right) \pi M_{\mathrm{K}}^{2}}{2 d^{2}\left(1-e^{2}\right)^{2}}+\frac{3 \pi a^{2}}{d^{2}\left(1-e^{2}\right)^{2}},
$$

where we have expanded the result of the integral also to the second order in $a$. Obviously this result reduces to the Schwarzschild one (see eq. (22)) for $a=0$, as expected.

\section{The rotating Chazy-Curzon particle}

A stationary generalization of the Curzon solution (4) representing a rotating Chazy-Curzon particle is given by the metric (27) with functions (see [12] and references therein)

$$
\begin{aligned}
& \psi_{\mathrm{C}_{\mathrm{rot}}}=\frac{1}{2} \ln \left[e^{2 \psi_{\mathrm{C}}} \frac{F_{1}}{F_{2}}\right], \quad \gamma_{\mathrm{C}_{\mathrm{rot}}}=\frac{1}{2} \ln \left[\frac{K_{1}}{16} e^{2 \gamma_{\mathrm{C}}} \frac{F_{1}}{R_{\mathrm{C}_{\mathrm{rot}}^{+}}^{+4} R_{\mathrm{C}_{\mathrm{rot}}}^{-4}}\right], \\
& w_{\mathrm{C}_{\mathrm{rot}}}=-k e^{-2 \psi_{\mathrm{C}}} \frac{F_{3}}{F_{1}}+K_{2}, \quad R_{\mathrm{C}_{\mathrm{rot}}}^{ \pm}=\sqrt{\rho^{2}+(z \pm k)^{2}},
\end{aligned}
$$

where $\psi_{\mathrm{C}}, \psi_{\mathrm{C}}$ refer to the seed solution (4), $F_{1}, F_{2}, F_{3}$ are functions of the coordinates $\rho$ and $z$

$$
\begin{aligned}
& F_{1}=\left\{16 R_{\mathrm{C}_{\mathrm{rot}}}^{+2} R_{\mathrm{C}_{\mathrm{rot}}}^{-2}+\left[\left(R_{\mathrm{C}_{\mathrm{rot}}}^{+}+R_{\mathrm{C}_{\mathrm{rot}}}^{-}\right)^{2}-4 k^{2}\right]^{2} a_{1} a_{2}\right\}^{2} \\
& +\left[\left(R_{\mathrm{C}_{\mathrm{rot}}}^{+}+R_{\mathrm{C}_{\mathrm{rot}}}^{-}\right)^{2}-4 k^{2}\right]\left[\left(R_{\mathrm{C}_{\mathrm{rot}}}^{+}-R_{\mathrm{C}_{\mathrm{rot}}}^{-}\right)^{2}-4 k^{2}\right]\left(R_{\mathrm{C}_{\mathrm{rot}}}^{+2} a_{1}-R_{\mathrm{C}_{\mathrm{rot}}}^{-2} a_{2}\right)^{2}, \\
& F_{2}=\left\{16 R_{\mathrm{C}_{\mathrm{rot}}}^{+2} R_{\mathrm{C}_{\mathrm{rot}}}^{-2}+\left[\left(R_{\mathrm{C}_{\mathrm{rot}}}^{+}+R_{\mathrm{C}_{\mathrm{rot}}}^{-}\right)^{2}-4 k^{2}\right]\left[R_{\mathrm{C}_{\mathrm{rot}}}^{+}+R_{\mathrm{C}_{\mathrm{rot}}}^{-}+2 k\right]^{2} a_{1} a_{2}\right\}^{2} \\
& +\left[R_{\mathrm{C}_{\mathrm{rot}}}^{+}+R_{\mathrm{C}_{\mathrm{rot}}}^{-}+2 k\right]^{2}\left\{-R_{\mathrm{C}_{\mathrm{rot}}}^{+2}\left[R_{\mathrm{C}_{\mathrm{rot}}}^{+}-R_{\mathrm{C}_{\mathrm{rot}}}^{-}-2 k\right] a_{1}\right. \\
& \left.+R_{\mathrm{C}_{\mathrm{rot}}}^{-2}\left[R_{\mathrm{C}_{\mathrm{rot}}}^{+}-R_{\mathrm{C}_{\mathrm{rot}}}^{-}+2 k\right] a_{2}\right\}^{2} \text {, } \\
& F_{3}=-R_{\mathrm{C}_{\mathrm{rot}}}^{+3} R_{\mathrm{C}_{\mathrm{rot}}}^{-3}\left[R_{\mathrm{C}_{\mathrm{rot}}}^{+}+R_{\mathrm{C}_{\mathrm{rot}}}^{-}+2 k\right]\left\{R_{\mathrm{C}_{\mathrm{rot}}}^{+}\left[R_{\mathrm{C}_{\mathrm{rot}}}^{+}-R_{\mathrm{C}_{\mathrm{rot}}}^{-}-2 k\right] a_{1}\right. \\
& +R_{\mathrm{C}_{\mathrm{rot}}}^{-}\left[R_{\mathrm{C}_{\mathrm{rot}}}^{+}-R_{\mathrm{C}_{\mathrm{rot}}}^{-}+2 k\right] a_{2}+\left[\left(R_{\mathrm{C}_{\mathrm{rot}}}^{+}+R_{\mathrm{C}_{\mathrm{rot}}}^{-}\right)^{2}-4 k^{2}\right] \\
& \times\left[R_{\mathrm{C}_{\mathrm{rot}}}^{+}+R_{\mathrm{C}_{\mathrm{rot}}}^{-}+2 k\right]^{2} a_{1} a_{2}\left\{\left[R_{\mathrm{C}_{\mathrm{rot}}}^{+}-R_{\mathrm{C}_{\mathrm{rot}}}^{-}-2 k\right]\left(a_{1} / R_{\mathrm{C}_{\mathrm{rot}}}^{-3}\right)\right. \\
& \left.\left.+\left[R_{\mathrm{C}_{\text {rot }}}^{+}-R_{\mathrm{C}_{\mathrm{rot}}}^{-}+2 k\right]\left(a_{2} / R_{\mathrm{C}_{\mathrm{rot}}}^{+3}\right)\right\}\right\} \text {, }
\end{aligned}
$$

with

$$
a_{1}=\alpha e^{-2\left[R_{\mathrm{C}_{\mathrm{rot}}}^{-} / R_{\mathrm{C}}-1\right]}, \quad a_{2}=\alpha e^{-2\left[R_{\mathrm{C}_{\mathrm{rot}}}^{+} / R_{\mathrm{C}}-1\right]},
$$


$R_{\mathrm{C}}$ is given in (4), and $K_{1}, K_{2}, k, \alpha$ are real constants, which are fixed by requiring a regular behaviour of the metric functions $\gamma_{\mathrm{C}_{\mathrm{rot}}}$ and $w_{\mathrm{C}_{\mathrm{rot}}}$ on the symmetry axis, in order to satisfy the condition of asymptotic flatness for the solution. They are expressed in terms of $k$ and $\alpha$, which define the total mass $M_{\mathrm{C}_{\text {rot }}}$ and the total angular momentum $J_{\mathrm{C}_{\mathrm{rot}}}$ of the rotating source

$$
M_{\mathrm{C}_{\mathrm{rot}}}=k \frac{1-3 \alpha^{2}}{1-\alpha^{2}}, \quad J_{\mathrm{C}_{\mathrm{rot}}}=2 \alpha k^{2} \frac{3-5 \alpha^{2}}{\left(1-\alpha^{2}\right)^{2}},
$$

as

$$
K_{1}=\frac{1}{\left(1-\alpha^{2}\right)^{2}}, \quad K_{2}=\frac{4 k \alpha}{1-\alpha^{2}} .
$$

Eqs. (35) - (39) fully determine the metric which could be used for the description of the exterior field of a stationary Curzon mass. When the rotation parameter $\alpha=0$, it reduces to the static Curzon solution (4). By requiring that the total mass $M_{\mathrm{C}_{\text {rot }}}$ be a positive quantity, one obtains the admissible values of the rotation parameter $\alpha$ :

$$
|\alpha|<\frac{1}{2}, \quad \text { and } \quad|\alpha|>1 .
$$

In the limit of small values of the rotation parameter, the relation (38) defining the total mass and angular momentum of the source becomes

$$
M_{\mathrm{C}_{\mathrm{rot}}} \simeq k, \quad J_{\mathrm{C}_{\mathrm{rot}}} \simeq 6 \alpha M_{\mathrm{C}_{\mathrm{rot}}}^{2},
$$

and the expected periastron shift results to be given by

$$
\Delta \phi_{\mathrm{C}_{\mathrm{rot}}} \simeq \frac{6 \pi M_{\mathrm{C}_{\mathrm{rot}}}}{d\left(1-e^{2}\right)}-\frac{96 \alpha \pi M_{\mathrm{C}_{\mathrm{rot}}}^{3 / 2}}{d^{3 / 2}\left(1-e^{2}\right)^{3 / 2}}+\frac{\left(44-9 e^{2}\right) \pi M_{\mathrm{C}_{\mathrm{rot}}}^{2}}{2 d^{2}\left(1-e^{2}\right)^{2}},
$$

where terms beyond the second order in $1 / d$ have been neglected, and reduces to the corresponding one for the static case (see eq. (22) ) for $\alpha=0$.

\section{Application: the S2-Sgr A* binary system}

Recently Ghez et al. 13 have observed a star orbiting close to the galactic center massive black hole. The star, which has been labelled as $\mathrm{S} 2$, with mass $M_{\mathrm{S} 2} \simeq 15 \mathrm{M}_{\odot}$, appears to be a main sequence star, orbiting the Galactic center black hole with a Keplerian period of $\simeq 15$ yrs. This has allowed 13 to estimate for the massive black hole in $\mathrm{SgrA}^{*}$ the mass of $M_{\mathrm{SgrA}} \simeq 4.07 \times 10^{6} M_{\odot}$. The orbital parameters of the S2-SgrA* binary system are listed in Table 1.

The relatively short orbital period of the $\mathrm{S} 2 \mathrm{star}$ allows in principle to easily attempt an observational campaign to look for genuine relativistic effects just like the orbital periastron shift. Since the amount of periastron advance strongly depends on the compactness of the central body, the detection of such an effect will give information about the nature of the dark object hosted in the center of the Galaxy. Hence, it becomes interesting to estimate the periastron shift for the S2-SgrA* binary system by assuming that the central gravitational field source is one of the object described in the previous sections. The results (expressed in arcseconds per revolution) are shown in Table 2 and 3 for fixed values of the parameters characterizing the solutions.

Obviously, the previous treatment also holds for different stars orbiting the galactic center region and for which the relativistic periastron advance effect might be clearer. In Figure 1 the expected 


\begin{tabular}{ll}
\hline \multicolumn{2}{c}{ S2-SgrA* ${ }^{*}$ orbital parameters } \\
\hline$M_{\mathrm{SgrA}}$ & $4.07 \times 10^{6} M_{\odot}$ \\
$M_{\mathrm{S} 2}$ & $15 M_{\odot}$ \\
$R_{\mathrm{S} 2}$ & $5.8 R_{\odot}$ \\
$d$ & $4.87 \times 10^{-3} \mathrm{pc}$ \\
$e$ & 0.87 \\
$P$ & $15.78 \mathrm{yr}$ \\
$i$ & $47.3 \mathrm{deg}$ \\
\hline
\end{tabular}

Table 1: The masses $M_{\mathrm{SgrA}}$ and $M_{\mathrm{S} 2}$ of the galactic center black hole and S2 orbiting star are given. The remaining orbital parameters are the $\mathrm{S} 2$ star radius $R_{\mathrm{S} 2}$, the semi-major axis $d$, the eccentricity $e$, the orbital period $P$ and the inclination angle $i$. Data are taken from Ghez et al. [13].

\begin{tabular}{|c|c|c|c|c|}
\hline$\Delta \phi$ (arcseconds/revolution) & $1 \mathrm{CC}$ & $1 \mathrm{~S}$ & $2 \mathrm{CC}$ & $2 \mathrm{~S}$ \\
\hline I order term & $6.5944 \times 10^{2}$ & $6.5944 \times 10^{2}$ & $6.5944 \times 10^{2}$ & $6.5944 \times 10^{2}$ \\
II order term & $3.4716 \times 10^{-1}$ & $3.2822 \times 10^{-1}$ & $2.2092 \times 10^{-1}$ & $2.1619 \times 10^{-1}$ \\
I + II order terms & $6.5986 \times 10^{2}$ & $6.5977 \times 10^{2}$ & $6.5966 \times 10^{2}$ & $6.5965 \times 10^{2}$ \\
\hline
\end{tabular}

Table 2: The periastron shifts (in arcseconds per revolution) corresponding to the single Chazy-Curzon particle, single Schwarzschild black hole, two Chazy-Curzon particles and two Schwarzschild black holes, respectively, are estimated in the case of the $\mathrm{S} 2-\mathrm{SgrA}^{*}$ binary system, whose orbital parameters are listed in Table 1 Here, we have assumed the S2 star orbital period to be 15.78 yrs. In the last two cases, the mass of each body has been taken equal to half of the total mass $M=M_{\mathrm{SgrA}}$ of the source, and the distance between them has been fixed equal to $b=3 M$. Note also that the second order term in these two cases can be made vanishing in correspondence of the choices $\tilde{b} / M \approx 9.96$ for $2 \mathrm{CC}$ and $\tilde{b} / M \approx 9.89$ for $2 \mathrm{~S}$.

periastron shift for an S2-like star as a function of the orbit eccentricity is shown assuming a central Schwarzschild black hole with mass $4.07 \times 10^{6} \mathrm{M}_{\odot}$. Dashed and solid lines are obtained for semimajor axes $d=10,5 \mathrm{mpc}$, respectively. The difference $\Delta$ between the expected single Schwarzschild black hole and the single Curzon particle periastron shifts (which is equal to that estimated for the corresponding two-body solutions, as from equations (23) and (25) is shown in Figure 2 Here, solid, dotted and dashed lines are obtained assuming $e=0.1,0.5$ and 0.9 , respectively. In Figure 3 instead, we show the expected periastron shift as a function of the orbital semi-major axis $d$, for fixed values of the orbit eccentricity $e=0.5$ and $e=0.87$. Solid and dashed lines correspond to the Schwarzschild and maximally Kerr $\left(a=M_{\mathrm{K}}\right)$ black holes cases, which for the chosen parameters represent the more favorable ones.

\section{Conclusions}

In this paper we have investigated the periastron position advance for various solutions belonging to the Weyl class of vacuum axially symmetric solutions to the Einstein field equations, namely those corresponding to a single Chazy-Curzon particle or Schwarzschild black hole as well as a pair of them 


\begin{tabular}{|c|c|c|}
\hline$\Delta \phi($ arcseconds/revolution $)$ & $1 \mathrm{~K}$ & 1 CCrot \\
\hline I order term & $6.5944 \times 10^{2}$ & $6.5944 \times 10^{2}$ \\
II order term & $2.3301 \times 10^{1}$ & $-2.2570 \times 10^{1}$ \\
I + II order terms & $6.8274 \times 10^{2}$ & $6.3687 \times 10^{2}$ \\
\hline
\end{tabular}

Table 3: The periastron shifts (in arcseconds per revolution) corresponding to the single Kerr black hole as well as to the single rotating Chazy-Curzon particle are estimated in the case of the S2-SgrA* binary system, with the choice of the parameter $a / M_{\mathrm{K}}=1$ and $\alpha=1 / 6$. In correspondence of this particular choice of the rotational parameters, the Kerr black hole and the rotating Chazy-Curzon particle are endowed with the same total angular momentum $J_{\mathrm{K}}=M^{2}=J_{\mathrm{C}_{\mathrm{rot}}}$.

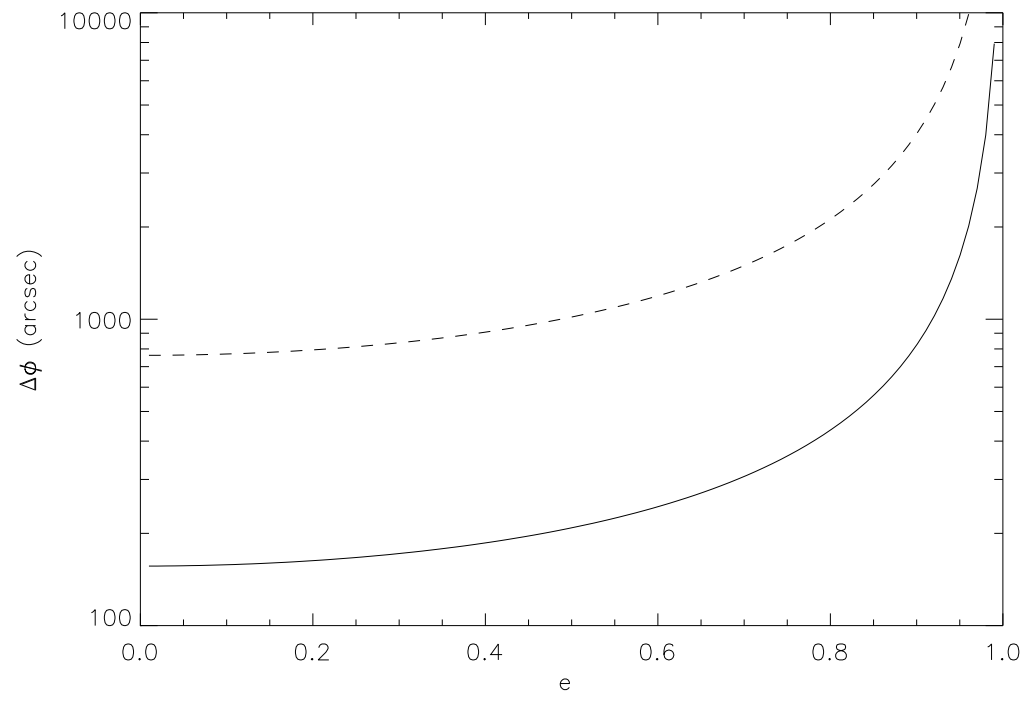

Figure 1: The expected periastron shift for an S2-like star as a function of the orbit eccentricity is shown assuming a central Schwarzschild black hole with mass $4.07 \times 10^{6} \mathrm{M}_{\odot}$. Dashed and solid lines are obtained for semi-major axes $d=10 \mathrm{mpc}$ and $d=5 \mathrm{mpc}$, respectively. 


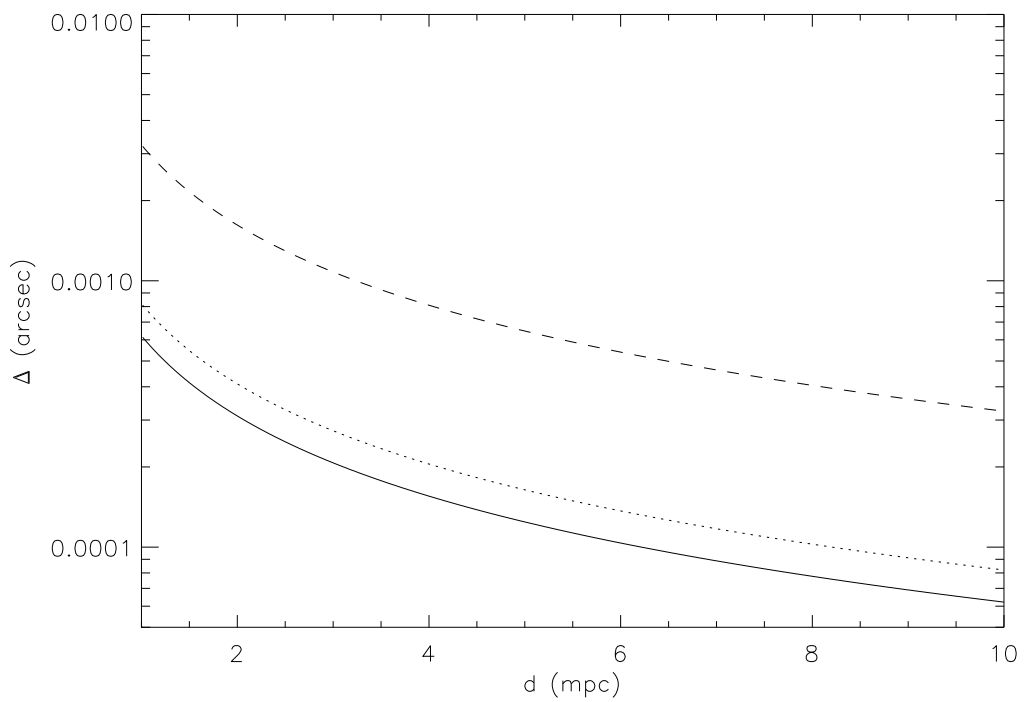

Figure 2: The difference $\Delta$ between the expected single Schwarzschild black hole and the single Curzon particle periastron shifts (which exactly coincides with that estimated for the corresponding two-body solutions) is shown as a function of the semi-major axis $d$. Here, solid, dotted and dashed lines are obtained assuming $e=0.1,0.5$ and 0.9 , respectively.

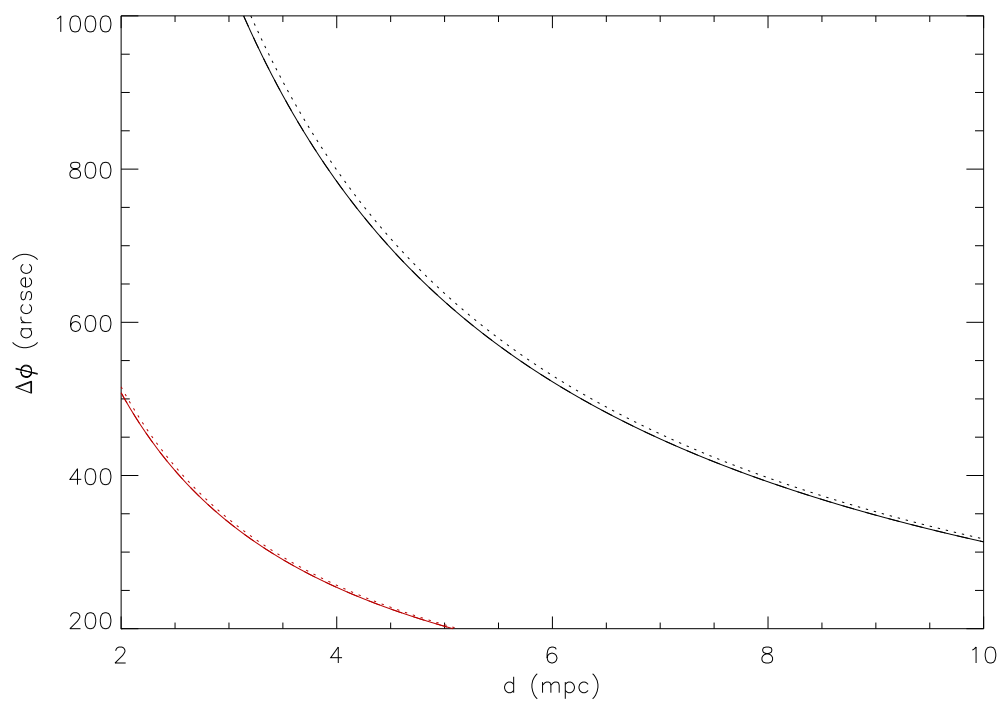

Figure 3: The expected periastron shift for an S2-like star as a function of the orbital semi-major axis is shown. Here, we have fixed the orbit eccentricity to $e=0.5$ (lower curves) and $e=0.87$ (upper curves). Solid and dashed lines correspond to the Schwarzschild and maximally Kerr $\left(a=M_{\mathrm{K}}\right)$ black holes cases. 
(static), and to a single rotating Chazy-Curzon particle or Kerr black hole (stationary). Although differences in the periastron shift among the considered solutions appear only at second order in the total mass $M$ of the central object, we have shown that the contribution of the second order term is appreciable with respect to the first order one, and can discriminate between the different configurations we have analyzed.

Our results are then applied to the physical system of the S2-SgrA* binary system at the galactic center. This research is particularly timely since within the next years it would be possible to definitely measure the periastron shift of the S2 star and therefore to univoquely determine, in principle, the nature of the dark object (usually assumed to be a black hole) hosted in the center of the Galaxy. However, a caution is needed since we expect that the galactic center dark object is surrounded by a compact stellar cluster which may gravitationally interact with the orbiting star producing an additional periastron shift which is retrograde with respect to the pure periastron shift predicted by the General Theory of Relativity. The effects of the presence of a stellar cluster at the galactic center and the constraints that present observations can put on its parameters (i.e. total mass, core radius and central matter density) will be discussed elsewhere.

\section{References}

[1] Chazy M., Bull. Soc. Math. France, 52, 17 (1924).

[2] Curzon H., Proc. London Math. Soc., 23, 477 (1924).

[3] Semerák O., Zellerin T. and Žáček M., MNRAS 308, 691 (1999).

[4] Semerák O., Žáček M. and Zellerin T., MNRAS 308, 705 (1999).

[5] Weyl H., Ann. Phys., Lpz. 54, 117 (1918).

[6] Letelier P.S. and Oliveira S.R., Class. Quantum Grav., 15, 421 (1998).

[7] Scott S.M. and Szekeres P., Gen. Relativ. Grav., 18, 557 (1986); Gen. Relativ. Grav., 18, 571 (1986).

[8] Sokolov D.D. and Starobinskii A.A., Sov. Phys. Dokl., 22, 312 (1977).

[9] Israel W., Phys. Rev., D15, 935 (1977).

[10] Bini D., Geralico A., Int. J. Mod. Phys. D, 13, 983 (2004).

[11] Stephani H., Kramer D., McCallum M.A.H., Hoenselaers C. and Hertl E., Exact solutions of Einstein's field equations, Cambridge University Press, Cambridge (1979).

[12] Hernández-Pastora J.L., Manko V.S. and Martín J., J. Math. Phys., 34, 4760 (1993).

[13] Ghez A.M., Duchêne G., Matthews K. et al., ApJ, 586, L127 (2003). 